\title{
ACCELERATION OF POLARIZED PROTONS TO HIGH ENERGY *
}

\author{
T. Roser ${ }^{\dagger}$, BNL, Upton, NY 11973, USA
}

\begin{abstract}
High energy polarized beam collisions will open up the unique physics opportunities of studying spin effects in hard processes. However, the acceleration of polarized beams in circular accelerators is complicated by the numerous depolarizing spin resonances. Using a partial Siberian Snake and a rf dipole that ensure stable adiabatic spin motion during acceleration has made it possible to accelerate polarized protons to $25 \mathrm{GeV}$ at the Brookhaven AGS. Full Siberian Snakes and polarimeters are being developed for RHIC to make the acceleration of polarized protons to 250 $\mathrm{GeV}$ possible. A similar scheme is being studied for the $800 \mathrm{GeV}$ HERA proton accelerator
\end{abstract}

\section{SPIN DYNAMICS, RESONANCES AND SIBERIAN SNAKES}

Accelerating polarized beams requires an understanding of both the orbital motion and spin motion. Whereas the effect of the spin on the orbit is negligible the effect of the orbit on the spin is usually very strong. The evolution of the spin direction of a beam of polarized protons in external magnetic fields such as exist in a circular accelerator is governed by the Thomas-BMT equation [1],

$$
\frac{d \vec{P}}{d t}=-\left(\frac{e}{\gamma m}\right)\left[G \gamma \overrightarrow{B_{\perp}}+(1+G) \overrightarrow{B_{\|}}\right] \times \vec{P}
$$

where the polarization vector $P$ is expressed in the frame that moves with the particle. This simple precession equation is very similar to the Lorentz force equation which governs the evolution of the orbital motion in an external magnetic field:

$$
\frac{d \vec{v}}{d t}=-\left(\frac{e}{\gamma m}\right)\left[\overrightarrow{B_{\perp}}\right] \times \vec{v} .
$$

From comparing these two equations it can readily be seen that, in a pure vertical field, the spin rotates $G \gamma$ times faster than the orbital motion. Here $G=1.7928$ is the anomalous magnetic moment of the proton and $\gamma=E / m$. In this case the factor $G \gamma$ then gives the number of full spin precessions for every full revolution, a number which also called the spin tune $\nu_{s p}$. At top RHIC energies this number reaches about 400 . The Thomas-BMT equation also shows that at low energies $(\gamma \approx 1)$ longitudinal fields $\overrightarrow{B_{\|}}$can be quite effective in manipulating the spin motion, but at high energies transverse fields $\overrightarrow{B_{\perp}}$ need to be used to have any effect beyond the always present vertical holding field.

- Work performed under the auspices of the U.S. Department of Energy

† Email: roser@bnl.gov

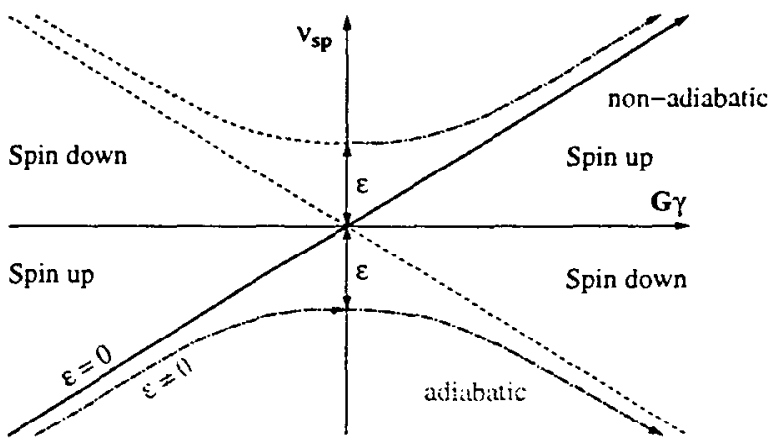

Figure 1: The evolution of the spin tune during the crossing of a resonance with strength $\epsilon$

The acceleration of polarized beams in circular accelerators is complicated by the presence of numerous depolarizing spin resonances. During acceleration, a spin resonance is crossed whenever the spin precession frequency equals the frequency with which spin-perturbing magnetic fields are encountered. There are two main types of spin resonances corresponding to the possible sources of such fields: imperfection resonances, which are driven by magnet errors and misalignments, and intrinsic resonances, driven by the focusing fields.

The resonance conditions are usually expressed in terms of the spin tune $\nu_{s p}$. For an ideal planar accelerator, where orbiting particles experience only the vertical guide field, the spin tune is equal to $G \gamma$, as stated earlier. The resonance condition for imperfection depolarizing resonances arise when $\nu_{s p}=G \gamma=n$, where $n$ is an integer. Imperfection resonances are therefore separated by only $523 \mathrm{MeV}$ energy steps. The condition for intrinsic resonances is $\nu_{s p}=G \gamma=k P \pm \nu_{y}$, where $k$ is an integer, $\nu_{y}$ is the vertical betatron tune and $P$ is the superperiodicity. For example at the AGS, $P=12$ and $\nu_{y} \approx 8.8$.

Close to a spin resonance the spin tune deviates away from its value of $G \gamma$ of the ideal flat machine. For a resonance with strength $\epsilon$, which is the total spin rotation due to the resonance driving fields, the new spin tune is given by the equation

$$
\cos \left(\pi \nu_{s p}\right)=\cos (\pi G \gamma) \cos (\pi \epsilon) .
$$

Fig. 1 shows the solutions of this equation with and without a resonance. A similar calculation can be done for the effective precession direction or, as it is now often called, the stable spin direction. The stable spin direction describes those polarization components that are repeated every turn. Note that both the stable spin direction and the spin tune are completely determined by the magnetic structure of the 
accelerator and the beam energy. The magnitude and sign of the beam polarization, however, depends on the beam polarization at injection and the history of the acceleration process.

The spin tune and stable spin direction calculations apply only to a time-independent static situation or if parameters are changed adiabatically. Far from the resonance the stable spin direction coincides with the main vertical magnetic field. Close to the resonance, the stable spin direction is perturbed away from the vertical direction by the resonance driving fields. When a polarized beam is accelerated through an isolated resonance at arbitrary speed, the final polarization can be calculated analytically [2] and is given by

$$
P_{f} / P_{i}=2 e^{-\frac{\pi|e|^{2}}{2 \alpha}}-1
$$

where $P_{i}$ and $P_{f}$ are the polarizations before and after the resonance crossing, respectively, and $\alpha$ is the change of the spin tune per radian of the orbit angle. When the beam is slowly ( $\alpha \ll|\epsilon|^{2}$ ) accelerated through the resonance, the spin vector will adiabatically follow the stable spin direction resulting in spin flip as is indicated in Fig. 1. However, for a faster acceleration rate partial depolarization or partial spin flip will occur.

Traditionally, the intrinsic resonances are overcome by using a betatron tune jump, which effectively makes $\alpha$ large, and the imperfection resonances are overcome with the harmonic corrections of the vertical orbit to reduce the resonance strength $\epsilon$ [3]. Both of these methods aim at making the resonance crossing non-adiabatic. They require very accurate adjustments at every resonance crossing which can become very difficult and time consuming.

Over the last ten years new techniques to cross both imperfection and intrinsic resonances adiabatically have been developed. The correction dipoles used to correct the imperfection resonance strength to zero were replaced by a localized spin rotator or 'partial Siberian snake' which makes all the imperfection resonance strengths large and causes complete adiabatic spin flip at every imperfection resonance [5]. The tune jump quadrupoles were recently replaced at the AGS by a single if dipole magnet which increased the strength of the intrinsic resonances by driving large coherent betatron oscillations.

At higher energies a 'full Siberian snake' [4], which is a $180^{\circ}$ spin rotator of the spin about a horizontal axis, will keep the stable spin direction unperturbed at all times as long as the spin rotation from the Siberian snake is much larger than the spin rotation due to the resonance driving fields. Therefore the beam polarization is preserved during acceleration. An alternative way to describe the effect of the Sibcrian snake comes from the observation that the spin tune with the snake is a half-integer and energy independent. Therefore, neither imperfection nor intrinsic resonance conditions can ever be met as long as the betatron tune is different from a half-integer.

A local spin rotator can be constructed by using either a solenoid at lower energies or at high energy by a sequence

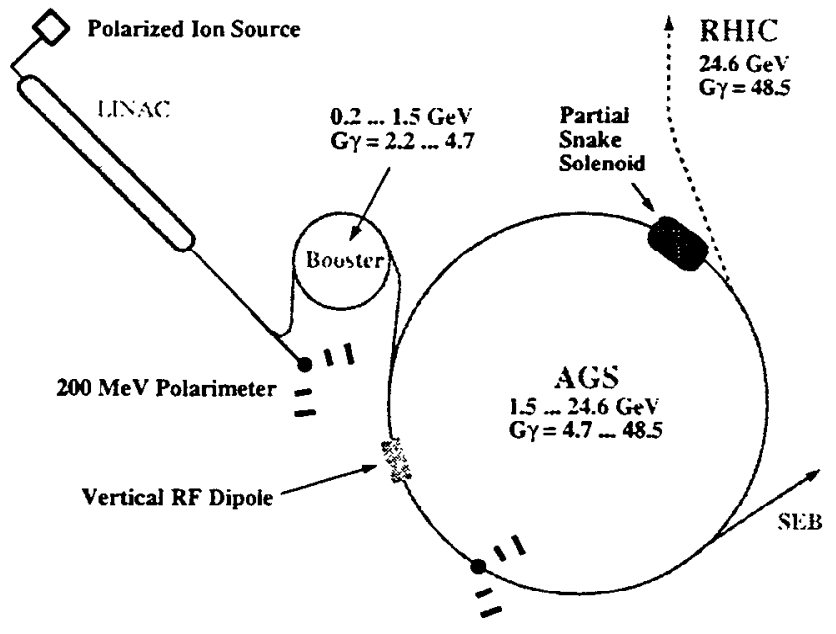

AGS Internal Polarimeter

Figure 2: Layout of the AGS accelerator complex showing the location of the partial Siberian snake, the vertical rf dipole, and the AGS internal polarimeter

of interleaved horizontal and vertical dipole magnets producing only a local orbit distortion. Since the orbit distortion is inversely proportional to the momentum of the particle, such a dipole snake is particularly effective for highenergy accelerators, e.g. energies above about $30 \mathrm{GeV}$.

\section{RECENT RESULTS FROM AGS, IUCF AND COSY}

Polarized proton beam experiments at the AGS have demonstrated the feasibility of polarized proton acceleration using a $5 \%$ partial Siberian snake. Fig. 2 shows a layout of the AGS accelerator complex highlighting the necessary hardware for polarized beam acceleration in the AGS. It was shown that a $5 \%$ snake is sufficient to avoid depolarization from imperfection resonances without using the harmonic correction method up to the required RHIC transfer energy of about $25 \mathrm{GeV}$. Fig. 3 shows the evolution of the beam polarization as the beam energy and therefore $G \gamma$ is increased [6]. As predicted the polarization reverses its sign whenever $G \gamma$ is equal to an integer. At this relatively low energy polarization is preserved even without snake but is partially lost at energies close to integer values of $G \gamma$.

More recently a novel scheme of overcoming strong intrinsic resonances using a if dipole magnet was successfully tested [8]. Full spin flip can be achieved with a strong artificial rf spin resonance excited coherently for the whole beam by driving large coherent vertical betatron oscillations. If the $\mathrm{rf}$ spin resonance location is chosen near the intrinsic spin resonance, the spin motion will be dominated by the $\mathrm{If}$ resonance and the spin near the intrinsic resonance will adiabatically follow the spin closed orbit of the of spin resonance. With the if dipole, a new dominant resonance near the intrinsic resonance is introduced to flip the spin, in- 


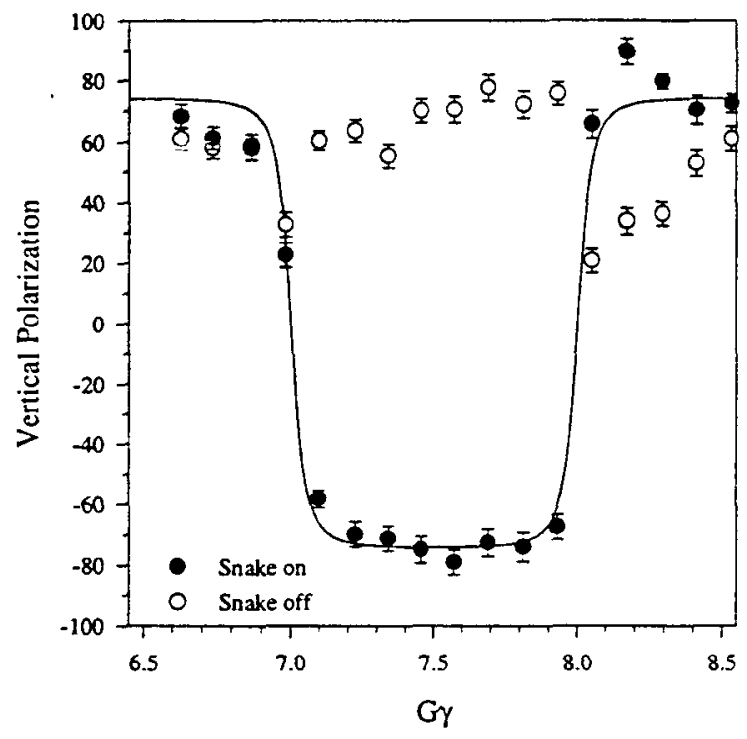

Figure 3: The measured vertical polarization as a function of $G \gamma$ for a $10 \%$ snake is shown with and without a snake. The solid line is the predicted energy dependence of the polarization.

stead of enhancing the intrinsic resonance, as has been proposed earlier [7], which would also enhance the strength of the nearby coupling resonance. Fig. 4 shows the new record proton beam polarization achieved during the last AGS polarized beam experiment. The rf dipole was used to completely flip the spin at the four strong intrinsic resonances $0+\nu_{y}, 12+\nu_{y}, 36-\nu_{y}$, and $36+\nu_{y}$. The lower curve shown going through the data points was obtained from a spin tracking calculation simulating the experimental conditions. Most of the remaining polarization loss is caused by the coupling resonances. A new AGS partial snake using a helical dipole magnet would eliminate all coupling resonances. Spin tracking simulations of this condition are depicted by the upper curve in Fig. 4.

During the last year the Cooler Synchrotron (COSY) at the Forschungszentrum in Juelich, Germany, has successfully accelerated polarized protons to $2.7 \mathrm{GeV} / \mathrm{c}$ [9]. With the luw acceleration rate used at COSY stable full spin flip at the few imperfection resonances can easily be achieved with small harmonic orbit distortions. The intrinsic resonances were crossed with a pulsed quadrupole using the tune jump method.

The studies of polarized proton acceleration and storage are also continuing at the IUCF Cooler. Most recently it was shown that an artificial spin resonance can be used to flip the spin of a store polarized beam even in the presence of a full Siberian snake[10]. In this as in all other experiments that use artificial spin resonances a simple oscillating field is used to drive the spin resonance. Even though an oscillating field is in fact the sum of two counter rotating fields, only one is generally in resonance with the beam precession frequency. However, with a full Siberian snake the spin tune is a half-integer and therefore the two

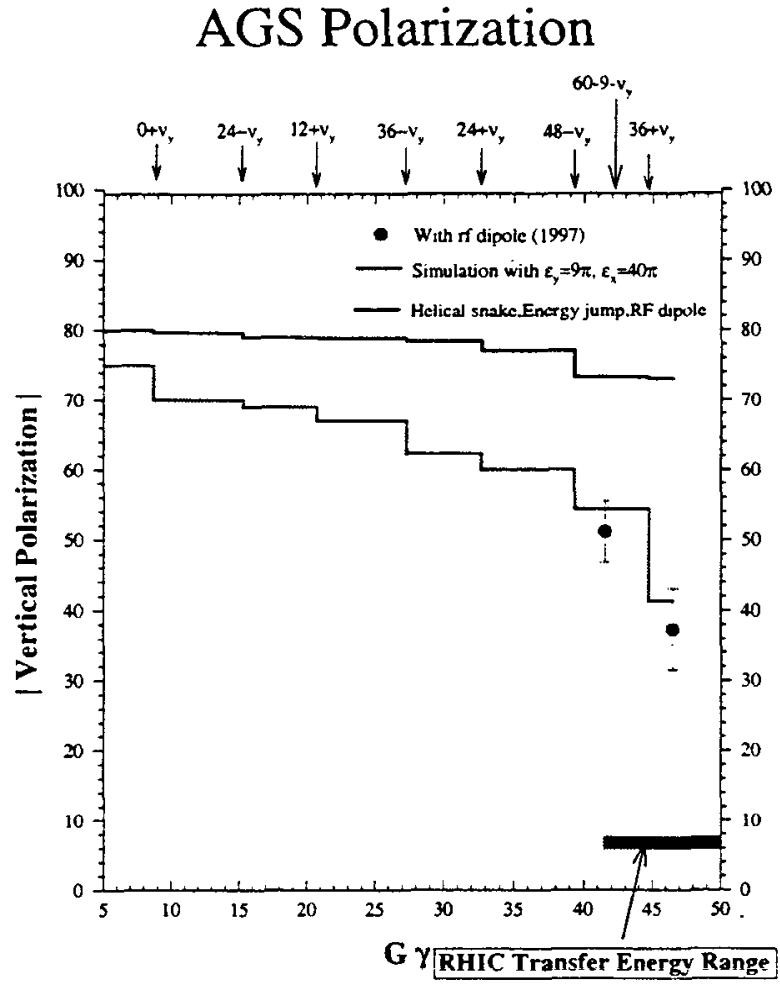

Figure 4: Vertical polarization versus $G \gamma$ measured in the AGS. The lower curve is the result of a spin tracking calculation for the experimental conditions. The upper curve simulates the use of a helical partial snake in the AGS.

counter-rotating fields are both in resonance and interfere so that effectively only half of the beam around the ring circumference sees a driving field. The IUCF experiment showed successfully that it is possible to slightly reduce the snake strength to avoid this degeneracy. A spin flip efficiency of $91 \%$ was achieved. This result is very encouraging for polarized proton colliders such as RHIC where the long storage times would make it necessary to reverse the polarization of the stored beam frequently to reduce systematic errors[11]. The efficiency reachable at the IUCF experiment is limited by the fact that both the snake and the rf resonance driving field are solenoids which can cause synchrotron sideband spin resonances close to the main artificial spin resonance. This would not be an issue at a high energy machine where transverse fields would be used for snakes and for driving spin resonances.

\section{PLANS FOR RHIC AND HERA}

By using Siberian snakes the stage is set for the acceleration of polarized proton beams to much higher energies. With snakes all depolarizing resonances should be avoided since the spin tune is a half-integer independent of energy. However, if the spin disturbance from small hori- 


\section{Polarized Proton Collisions at BNL}

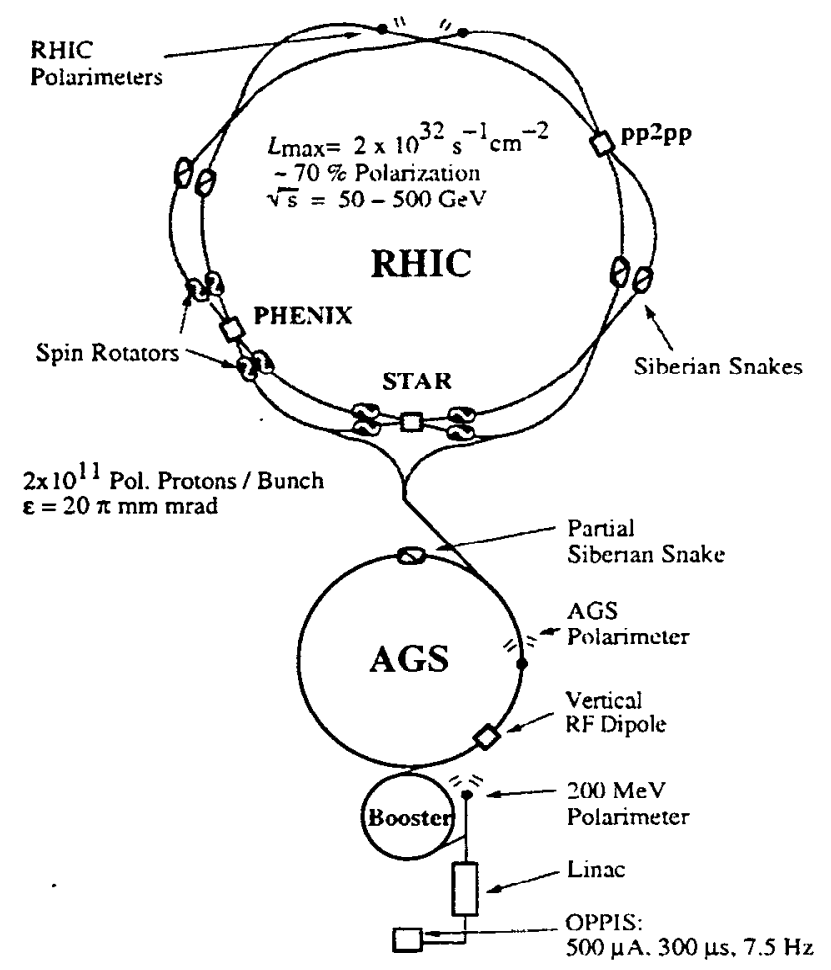

Figure 5: The Brookhaven hadron facility complex, which includes the AGS Booster, the AGS, and RHIC. The RHIC spin project will install two snakes per ring with four spin rotators per detector for achieving helicity-spin experiments.

zontal fields is adding up sufficiently between the snakes depolarization can still occur. This is most pronounced when the spin rotation from all the focusing fields add up coherently which is the case at the strongest intrinsic resonances. A simplistic rule of thumb would then suggest that as long as the total spin rotation of all the Siberian snakes is much larger than the total spin rotation per turn caused by the strongest spin resonance the polarization should be preserved during acceleration. This rule hc lds for the AGS partial Siberian snake with regard to the imperfection resonances. It would also predict that for a beam with a normalized $95 \%$ emittance of $20 \pi \mathrm{mm} \mathrm{mrad}$ at least two snakes are needed for RHIC and four snakes for HERA.

Polarized protons from the AGS are injected into the two RHIC rings to allow for up to $\sqrt{s}=500 \mathrm{GeV}$ collisions with both beams polarized [12]. Fig. 5 shows the lay-out of the Brookhaven accelerator complex highlighting the components required for polarized beam acceleration.

Of particular interest is the design of the Siberian snakes (two for each ring) and the spin rotators (four for each collider experiment) for RHIC. Each snake or spin rotator consists of four $2.4 \mathrm{~m}$ long, $4 T$ helical dipole magnet modules each having a full 360 degree helical twist [13]. Using helical magnets minimizes orbit excursions within the extend

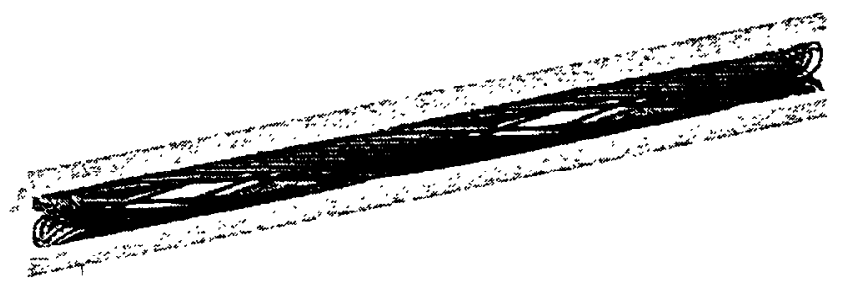

Figure 6: Schematic picture of the superconducting helical dipole shows the 16 helical current blocks and half of the iron yoke

of the snake or spin rotator which is most important at injection energy. Nevertheless the bore of the helical magnets has to be $10 \mathrm{~cm}$ in diameter to accommodate the $3 \mathrm{~cm}$ orbit excursions. Superconducting helical dipoles have been successfully tested at BNL using thin cable placed into helical grooves that have been milled into a thick-walled aluminum cylinder. A schematic picture of the helical dipole magnet is shown in Fig. 6. The first full length magnet has recently been successfully tested[14].

To verify that full polarization is preserved during acceleration in RHIC an elaborate spin tracking program was developed[15]. The acceleration through the energy region of the strongest resonance was simulated in great detail including a $1 \mathrm{~mm} \mathrm{rms}$ misalignment of the quadrupoles, and sextupoles as well as the corrector dipoles used to correct the closed orbit. The result is shown in Fig. 7 for a beam with a normalized $95 \%$ emittance of $20 \pi \mathrm{mm} \mathrm{mrad}$. The upper and lower curve show the result for the full beam and the particles at the edge of the beam, respectively. Although there is a significant decrease of the polarization at the energy of the resonance at $G \gamma=5 \times 81+\left(\nu_{y}-12\right)=$

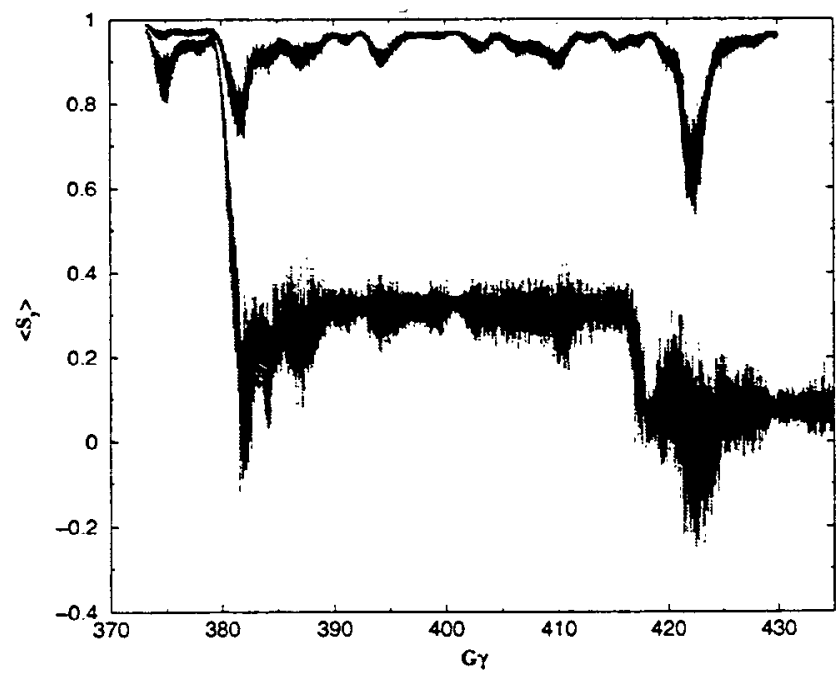

Figure 7: Result of spin tracking through the region of the strongest resonance in RHIC. The closed orbit was corrected with residuals of less than $0.2 \mathrm{~mm}$. 
422.18 , the polarization of the full beam is restored after accelerating completely through the resonance region. The simulation also shows that there is significant polarization loss at the edge of the beam. This fact highlights the need for a polarimeter that can measure polarization profiles.

Measuring proton polarization at high energies has been the focus of several workshops and experiments. The analyzing power of only very few reactions has been measured at high energies and the magnitudes are typically rather small. Polarization sensitive interaction with an external electromagnetic field is also much smaller than for the much lighter electron for which Compton back scattering is typically used for high energy polarization measurement. Two methods are presently being considered for RHIC. Inclusive pion production from both hydrogen and carbon targets has been shown to have large analyzing powcr. Magnetic spectrometers are being designed for RHIC to allow for high rate data acquisition. The second method is based on the relatively large, energy independent analyzing power predicted for very small angle elastic scattering where the magnetic interaction interferes with the spin independent nuclear interaction[16]. Tests are presently in progress to demonstrated the possibility of detecting the recoil carbon nucleus from a ultra thin carbon fiber. For both methods the carbon fiber target could be scanned through the circulating bearn to measure polarization profiles.

The HERA collider consists of a $30 \mathrm{GeV}$ electron ring and a $820 \mathrm{GeV}$ proton ring. Electron beam polarization of about $70 \%$ has been achieved and spin rotators are successfully being used to produce longitudinal polarization for the HERMES fixed target experiment. Recently, there is interest to examine the possibility to also polarize the HERA proton beam [17]. With a top energy of $820 \mathrm{GeV}$ the acceleration of polarized protons in HERA would need at least four snakes. However the high energy will require very detailed studies to determine the beam emittance and magnet alignments that are required to successfully accelerate polarized protons. HERA faces an additional complication from the vertical excursions that were included at the interaction regions with the electron beam. Additional snakes are needed to make these excursions spin transparent.

\section{CONCLUSIONS}

With all the recent advances in the understanding of spin dynamics and the development of techniques for spin manipulation, polarized beam operation could become more of an integral part of future high energy accelerators. In particular, polarized proton beam capabilities are being developed for RHIC It is anticipated that, for the expected turn-on of RHIC late in 1999, one ring will be equipped with two snakes and a polarimeter which will allow for initial commissioning of polarized beam acceleration. The remaining snakes and all the spin rotators for the two detectors STAR and PHENIX will be in place for the first RHIC spin physics run starting after October 2000.

\section{REFERENCES}

[1] L.H. Thomas, Phil. Mag. 3, 1 (1927); V. Bargmann, L. Michel, V.L. Telegdi, Phys. Rev. Lett. 2, 435 (1959).

[2] M. Froissart and R. Stora, Nucl. Instr. Meth., 1, 297 (1960).

[3] T. Khoe et al., Part. Accel. 6, 213 (1975); J.L. Laclare et al., J. Phys. (Paris), Collog. 46, C2-499 (1985); H. Sato et al., Nucl. Inst. Meth., Phys. Res. Sec A272, 617 (1988); F.Z. Khiari, et al., Phys. Rev. D39, 45 (1989).

[4] Ya.S. Derbenev et al., Part. Accel. 8, 115 (1978).

[5] T. Roser, AIP Conf. Proc. No. 187, ed. K.J. Heller p.1442 (AIP, New York, 1988).

[6] H. Huang et al., Phys. Rev. Lett. 73, 2982 (1994)

[7] T. Roser, in Proc. of the 10th Int. Symp. on High Energy Spin Physics, Nagoya, Japan, p. 429 (1992).

[8] M. Bai et al., Phys. Rev. Lett. 80, 4673 (1998)

[9] A. Lehrach, Ph.D. thesis, Forschungszentrum Juelich, Germany, 1997

[10] D.D. Caussyn et al., Phys. Rev. Lett. 73, 2857 (1994), B.B. Blinov et al., Phys. Rev. Lett. 81, 2906 (1998)

[11] M. Bai, 'Beam manipulations with an of dipole', these proceedings; B. Parker et al., 'Design of an ac-dipole for use in RHIC', these proceedings.

[12] Design Manual - Polarized Proton Collider at RHIC, Brookhaven National Laboratory, July 1998, http://www.ags.bnl.gov/ rhicspin .

[13] V.I.Ptitsin and Yu.M.Shatunov, Helical Spin Rotators and Snakes, Proc. 3. Workshop on Siberian Snakes and Spin Rotators (A.Luccio and T.Roser Eds.) Upton, NY, Sept. 1213,1994, BNL-52453, p.15;

[14] E. Willen et al., 'Construction of helical magnets for RHIC', these proceedings.

[15] A.U. Luccio et al., 'Development of the spin tracking program SPINK', these proceedings.

[16] J. Schwinger, Phys. Rev. 73, 407 (1948)

[17] 'Acceleration of Polarized Protons to $820 \mathrm{GeV}$ at HERA', University of Michigan Report HE 96-20, 1996 